\title{
Assessing Near-Future Direct Dark Matter Searches with Benchmark-Free Forecasting
}

\author{
Thomas D. P. Edwards, ${ }^{*}$ Bradley J. Kavanagh, ${ }^{\dagger}$ and Christoph Weniger ${ }^{*}$ \\ Gravitation Astroparticle Physics Amsterdam (GRAPPA), Institute for Theoretical Physics Amsterdam \\ and Delta Institute for Theoretical Physics, University of Amsterdam, \\ Science Park 904, 1090 GL Amsterdam, Netherlands
}

(Received 24 May 2018; published 2 November 2018)

\begin{abstract}
Forecasting the signal discrimination power of dark matter (DM) searches is commonly limited to a set of arbitrary benchmark points. We introduce new methods for benchmark-free forecasting that instead allow an exhaustive exploration and visualization of the phenomenological distinctiveness of DM models, based on standard hypothesis testing. Using this method, we reassess the signal discrimination power of future liquid xenon and argon direct DM searches. We quantify the parameter regions where various nonrelativistic effective operators, millicharged DM, and magnetic dipole DM can be discriminated, and where upper limits on the DM mass can be found. We find that including an argon target substantially improves the prospects for reconstructing the DM properties. We also show that only in a small region with DM masses in the range 20-100 GeV and DM-nucleon cross sections a factor of a few below current bounds can near-future xenon and argon detectors discriminate both the DM-nucleon interaction and the DM mass simultaneously. In all other regions only one or the other can be obtained.
\end{abstract}

DOI: $10.1103 /$ PhysRevLett.121.181101

Introduction.-Searching for the elusive dark matter (DM) particle has been the preoccupation of physicists for many years $[1,2]$. Over the past decade, two-phase scintillator direct detection experiments $[3,4]$ have found much success, with the LUX [5], XENON [6], and PANDA-X [7] Collaborations providing the most stringent constraints on DM particles in the GeV-TeV mass range to date. Such experiments will continue to improve in sensitivity for many years to come. In the case of a detection, it should be possible to study the astro- and particle-physics properties of DM using a variety of detectors and detection methods (see [8-12] and many others), but the precise parameter regions in which these properties can actually be measured is hard to quantify.

Exploring the prospects for discriminating between different DM-nucleon interactions usually relies on comparing a number of benchmark models [13-19]. However, the pair-wise comparison of different benchmark points in the model parameter space (DM couplings or masses) is time consuming, does not scale well with the number of benchmark points, and is in particular problematic in high-dimensional parameter spaces. In direct detection, such a high-dimensional parameter space appears in the

Published by the American Physical Society under the terms of the Creative Commons Attribution 4.0 International license. Further distribution of this work must maintain attribution to the author(s) and the published article's title, journal citation, and DOI. Funded by SCOAP . framework of nonrelativistic effective field theory (NREFT) [20-24], in which the space of DM-nucleon interactions may have more than 30 dimensions [25-27]. With current techniques, it is hence difficult to study model degeneracies and the degeneracy-breaking power of future instruments in a reliable and exhaustive way. For such tasks, dedicated techniques are required.

In this Letter, we introduce a new framework for studying the signal discrimination power of future detectors in a fundamentally benchmark-free way. The key questions we aim to address are these: how many observationally distinct signals does a given model predict for a set of future experiments? How many of these signals are compatible with specific subsets of the signal model? In which regions of parameter space is signal discrimination and parameter reconstruction possible?

We first summarize the basics of our approach. We then discuss the dark matter models and experiments we consider in the current Letter. Finally, we show our results and conclude with a short discussion about possible future directions and applications [28].

Information Geometry.-Consider a new-physics model $\mathcal{M}$ with some $d$-dim model parameter space, $\vec{\theta} \in \Omega_{\mathcal{M}} \in \mathbb{R}^{d}$, and a combination of future experiments $X$ that are described by some likelihood function $\mathcal{L}_{X}(\mathcal{D} \mid \vec{\theta})$, where $\mathcal{D}$ is data. We expect that two model parameter points $\vec{\theta}, \vec{\theta}^{\prime}$ can be discriminated by experiments $X$ if the parameter point $\vec{\theta}^{\prime}$ is inconsistent with Asimov data [29] $\mathcal{D}=\overline{\mathcal{D}}(\vec{\theta})$. More concretely, distinctiveness requires that the log-likelihood ratio, 


$$
\operatorname{TS}\left(\vec{\theta}^{\prime}\right)_{\overline{\mathcal{D}}(\vec{\theta})} \equiv-2 \ln \frac{\mathcal{L}\left(\overline{\mathcal{D}}(\vec{\theta}) \mid \vec{\theta}^{\prime}\right)}{\max _{\vec{\theta}^{\prime \prime}} \mathcal{L}\left(\overline{\mathcal{D}}(\vec{\theta}) \mid \vec{\theta}^{\prime \prime}\right)} \simeq\left\|\vec{x}(\vec{\theta})-\vec{x}\left(\vec{\theta}^{\prime}\right)\right\|^{2}
$$

exceeds a threshold value $r_{\alpha}(\mathcal{M})^{2}$. The threshold value depends on the chosen statistical significance, which we set here to $\alpha=0.045(2 \sigma)$, as well as the sampling distribution of $\operatorname{TS}\left(\vec{\theta}^{\prime}\right)$. In the large-sample limit and under certain regularity conditions, the sampling distribution follows a $\chi_{k}^{2}$ distribution with $k=d$ degrees of freedom [29,30].

The last part of Eq. (1) is an approximation based on the "Euclideanized signal" method [31], an embedding $\vec{\theta} \mapsto \vec{x}(\vec{\theta}) \in \mathbb{R}^{n}$ into some, usually higher-dimensional, space with unit Fisher information matrix ( $n$ usually equals the total number of data bins). This approximation maps statistical distinctiveness onto Euclidean distances, and works to within $20 \%$ if the number of counts is order 1 , see Ref. [31] for a discussion and caveats. Confidence regions in the model parameter space correspond then to hyperspheres of the radius $r_{\alpha}(\mathcal{M})$ in the Euclideanized signal space.

Often one is interested in submodels $\mathcal{S}$ that are nested inside model $\mathcal{M}$, and which are obtained by restricting $\mathcal{M}$ to a $d^{\prime}$-dim subregion $\Omega_{\mathcal{S}} \subset \Omega_{\mathcal{M}}$. A parameter point $\vec{\theta}$ of $\mathcal{M}$ leads to a signal that is distinct from any signal in submodel $\mathcal{S}$ if

$$
-2 \ln \frac{\max _{\vec{\theta}^{\prime} \in \Omega_{\mathcal{S}}} \mathcal{L}\left(\overline{\mathcal{D}}(\vec{\theta}) \mid \vec{\theta}^{\prime}\right)}{\max _{\vec{\theta}^{\prime}} \mathcal{L}\left(\overline{\mathcal{D}}(\vec{\theta}) \mid \vec{\theta}^{\prime}\right)} \simeq \min _{\vec{\theta}^{\prime} \in \Omega_{\mathcal{S}}}\left\|\vec{x}(\vec{\theta})-\vec{x}\left(\vec{\theta}^{\prime}\right)\right\|^{2}
$$

exceeds a certain threshold value $r_{\alpha}(\mathcal{S}, \mathcal{M})^{2}$. Here "distinct" means that the composite null hypothesis $\mathcal{S}$ can be rejected for data $\overline{\mathcal{D}}(\vec{\theta})$. The sampling distribution of Eq. (2) follows in general a $\chi_{k=d-d^{\prime}}^{2}$ distribution. In the Euclideanized signal space $\vec{x}$, parts of model $\mathcal{M}$ cannot be discriminated from model $\mathcal{S}$ that lie within a "shell" of thickness $r_{\alpha}(\mathcal{S}, \mathcal{M})$ around the signal manifold of $\mathcal{S}$.

Finally, nuisance parameters can be accounted for by replacing the likelihood function in Eq. (1) with a profile likelihood, $\mathcal{L}(\mathcal{D} \mid \vec{\theta})=\max _{\vec{\eta}} \mathcal{L}(\mathcal{D} \mid \vec{\theta}, \vec{\eta}) \mathcal{L}_{\eta}(\vec{\eta})$, where the last factor can incorporate additional constraints on the nuisance parameters from data external to $X$.

Distinct signals.-To quantify the signal discrimination power of a set of future experiments $X$ in the context of model $\mathcal{M}$ we may define the figure of merit

$$
\nu_{\mathcal{M}, X}^{\alpha}\left(\Omega_{\mathcal{M}}\right) \simeq \begin{aligned}
& \text { Total number of signals from model } \mathcal{M} \\
& \text { discriminable by experiments } X .
\end{aligned}
$$

More specifically, $\nu_{\mathcal{M}, X}^{\alpha}$ equals the maximum number of points that can populate the parameter space of $\mathcal{M}$ while remaining mutually distinct according to Eq. (1). Any such

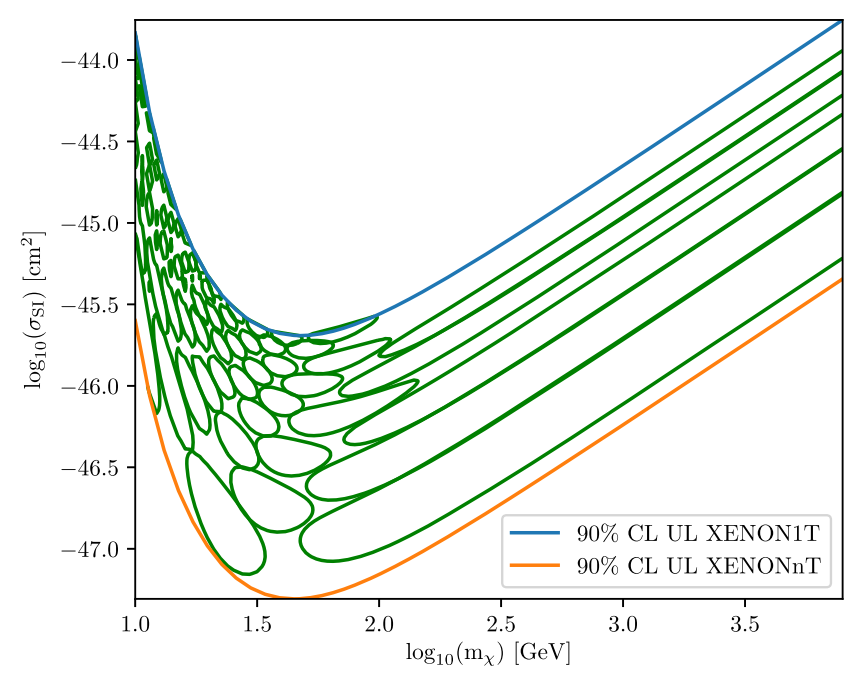

FIG. 1. Limit on the SI cross section vs DM mass, assuming operator $\mathcal{O}_{1}$, for a XENONnT detector. Contours show $68 \%$ confidence contours in $d=2$ dimensions. The radius of these contours in the Euclidean space is therefore $r_{\alpha}(\mathcal{M})=1.52$. The number of discriminable signals in the blue + blue-orange region of the middle panel of Fig. 2 can be approximated by counting the number of closed green contours.

set of points provides a complete sample of the phenomenological features of model $\mathcal{M}$. Loosely speaking, the points correspond to a set of nonoverlapping confidence contours as shown in Fig. 1. Furthermore, when considering a submodel $\mathcal{S}$ nested in $\mathcal{M}$, we can define

$$
\begin{aligned}
\nu_{\mathcal{M}, X}^{\alpha}\left(\Omega_{\mathcal{S}}\right)= & \begin{array}{l}
\text { Number of signals from model } \mathcal{M} \\
\text { discriminable by experiments } X, \text { and } \\
\text { consistent with model } \mathcal{S} .
\end{array}
\end{aligned}
$$

With these definitions, the phenomenological distinctiveness of various regions in the parameter space of model $\mathcal{M}$ can be visualized using standard Venn diagrams [32]. The technical definition for the measure $\nu_{\mathcal{M}, X}^{\alpha}(\cdot)$, which is used in the subsequent examples, is given in Appendix A of the Supplemental Material [33].

DM-nucleon interactions. - While direct detection is typically analyzed in terms of the standard spin-dependent (SD) and spin-independent (SI) interactions [34], the range of possible signals is much broader. The framework of nonrelativistic effective field theory (NREFT) [20-24] aims to classify possible elastic DM-nucleon interactions and thus possible signals in DM-nucleus scattering experiments. NREFT is realized as a power series in the DM-nucleus relative velocity $\vec{v}$ and the nuclear recoil momentum $\vec{q}$, valid for nonrelativistic, short-range interactions. The resulting operators (labeled $\mathcal{O}_{1}, \mathcal{O}_{3}, \mathcal{O}_{4}, \ldots$ ) give rise to a range of novel energy spectra $[14,16,19,35]$, directional signals [36,37], and annual modulation signatures $[38,39]$. We focus here on the three operators $\mathcal{O}_{1}, \mathcal{O}_{4}$, 
and $\mathcal{O}_{11}$ because they allow us to explore a diverse range of signals with only a small number of operators [40]. Operator $\mathcal{O}_{1}=1_{\chi} 1_{N}$ couples to nucleon number while the operator $\mathcal{O}_{4}=\vec{S}_{\chi} \cdot \vec{S}_{N}$ couples to nuclear spin, allowing us to explore the complementarity between nuclei of different size and spin [43]. Operator $\mathcal{O}_{11}=i \vec{q} \cdot \vec{S}_{\chi} / m_{N}$ may arise as the leading-order interaction in certain scalarmediated models [24]. Similar to $\mathcal{O}_{1}$, it also couples to nucleon number and receives a coherent enhancement to the rate, but has a characteristic peaked recoil spectrum owing to an extra $d \sigma / d E_{R} \propto E_{R}$ scaling of the cross section [16]. This allows us to explore how well different recoil spectra can be discriminated in future experiments.

Unfortunately, NREFT cannot encompass all possible signals. In particular, in its original formulation [21], it cannot describe interactions through light mediators. In this case, the typical momentum transfer is larger than the mediator mass and an expansion in $q$ is no longer appropriate [44]. The scenario in which this mediator is the standard model photon has been studied extensively $[45,47,48]$. Here, we consider millicharged DM [49], which has long-ranged, coherently enhanced interactions with nuclei, with a differential cross section scaling as $E_{R}^{-2}$ [45,50,51]. Alternatively, DM may have nonzero electric and magnetic moments [52,53], particularly if it takes the form of a composite state, such as a Dark Baryon [54,55]. In the context of model discrimination, most interesting for us will be the magnetic dipole, $\left(\mu_{\chi} / 2\right) \bar{\chi} \sigma^{\mu \nu} \chi F_{\mu \nu}$, which leads to both long-range and short-range contributions to the rate, arising from charge-dipole and dipole-dipole interactions, respectively $[48,56,57]$.

The five DM-nucleon interaction models that we have outlined above encompass a range of phenomenologically driven as well as more theoretically motivated models, leading to a wide range of direct detection signals. We calculate the signal spectra in each case using the publicly-available code WIMPY [58], implementing expressions from [23,45] (see Ref. [59] for more details). The required nuclear response functions are taken from the Mathematica package provided in [23], supplemented by those calculated in [60]. We assume isospin conserving $\left(c_{p}=c_{n}\right)$ NREFT interactions and that the particle producing a signature makes up $100 \%$ of the local DM density (which we fix to $\rho_{\chi}=0.3 \mathrm{GeV} \mathrm{cm}^{-3}[61,62]$ ). We incorporate standard Gaussian halo uncertainties from [62]; details can be found in Appendix B of the Supplemental Material [33].

Direct dark matter searches.-We implement two toy detectors, designed to resemble the expected advancement in direct DM searches over the upcoming 5-10 years.

We implement a xenon detector in light of the stringent constraints on $\mathcal{O}(10) \mathrm{GeV}$ DM set by XENON1T [6], with numerous xenon-based experiments on the horizon [63-66]. We model this detector on the future XENONnT [65] experiment. In addition we implement a detector containing a target material with no nuclear spin, namely argon, modeling this detector on Darkside20k [67]. In this way we maximize discriminability of spin-dependent operators [68]. Our detector implementations and background assumptions are briefly described in Appendix B of the Supplemental Material [33].

Results.-In Fig. 1, we show the expected $68 \%$ CL reconstruction regions for a set of mutually distinct parameter points, for our XENONnT-like detector. The confidence regions are constructed by querying spheres with radius $r_{\alpha}(\mathcal{M})=1.52$ in the Euclideanized signal space. The number of these regions corresponds approximately to the figure of merit in Eq. (3).

In the central panel of Fig. 2, we illustrate the power of XENONnT to discriminate between operators in the threedimensional model space $\mathcal{M}$ of mass, $\mathcal{O}_{1}$, and $\mathcal{O}_{11}$. With increasing numbers of events, the number of discriminable signals increases, though the majority of signals are
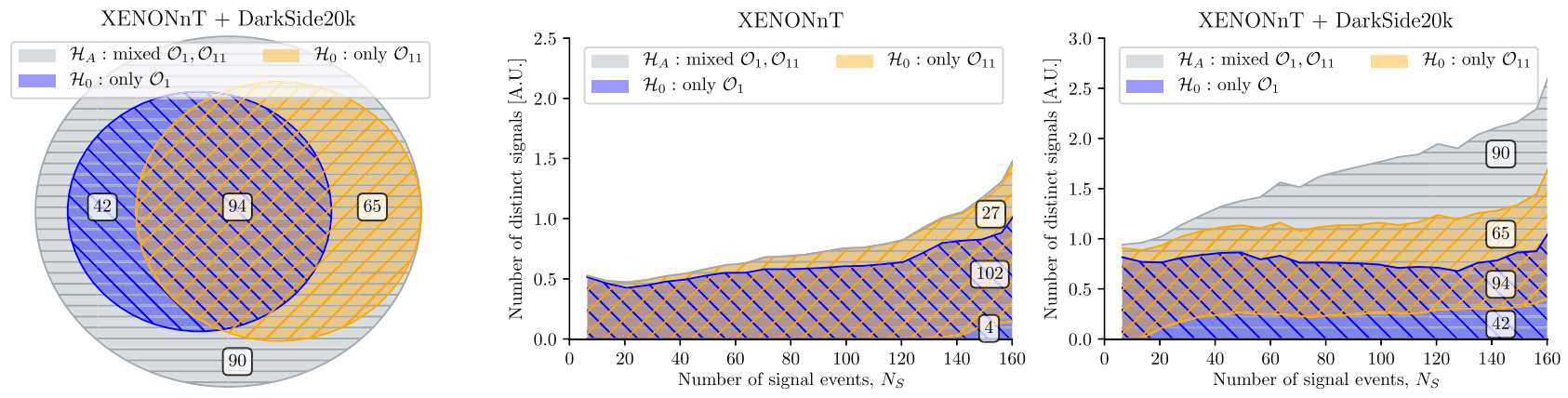

FIG. 2. Signal-discrimination power of combinations of direct detection experiments, summarized in infometric Venn diagrams. Central-Right panel: the full area corresponds to the number of distinct detectable signals within the alternative hypothesis $\mathcal{H}_{A}$, unfolded as function of number of XENONnT signal events. The subsets indicate the fraction of signals consistent with various null hypotheses $\mathcal{H}_{0}$. Overlapping subsets correspond to signals consistent with multiple null hypotheses simultaneously. The numbers correspond to $\nu_{\mathcal{M}, X}\left(\Omega_{\mathcal{M}}\right)$ in each region. In the right panel, the overlapping (blue + orange) region corresponds to the model parameters between the purple-dot-dashed and orange contours in Fig. 3. The nonoverlapping (blue only) $\mathcal{O}_{1}$ region corresponds to parameters between the purple-dashed and blue contours in Fig. 3. Left panel: standard Venn diagram summed over number of signal events. 
compatible with both $\mathcal{O}_{1}$ and $\mathcal{O}_{11}$. In the right panel of Fig. 2, we include also information from DarkSide20k. The addition of an argon detector not only increases the number of discriminable signals (from 133 to 291) but also enlarges the region of parameter space where $\mathcal{O}_{1}$ and $\mathcal{O}_{11}$ can be discriminated from each other. The left panel of Fig. 2 corresponds to the same scenario as the right panel, instead summed over the number of XENONnT signal events: 160 signal events approximately corresponds the expected number of events in XENON-nT if the true model were at the current sensitivity. We note that the Venn diagrams we have introduced here significantly increase in complexity when comparing a large number of models at once. However, we emphasize that the number of discriminable regions, Eq. (3), is completely general and remains a useful measure for assessing model discriminability.

Figure 3 shows the regions of the parameter space of spin-independent $\left(\mathcal{O}_{1}\right)$ DM in which discrimination from $\mathcal{O}_{4}, \mathcal{O}_{11}$, and magnetic dipole DM would be possible. For $\mathcal{O}_{4}$ (spin dependent), $2 \sigma$ discrimination is possible at high DM mass even down to small cross sections, when both xenon and argon experiments are used. The spin-zero argon nucleus has no spin-dependent coupling, so we can discriminate well as long as the argon detector has sensitivity $\left[m_{\chi}>\mathcal{O}(20 \mathrm{GeV})\right.$, below which most recoils are below the $32 \mathrm{keV}$ threshold].

For $\mathcal{O}_{11}$, discrimination is possible at high mass because of the different spectral shapes of $\mathcal{O}_{1}$ and $\mathcal{O}_{11}$, though cross sections around $\sim 10^{-46} \mathrm{~cm}^{2}$ are required to obtain enough events to map out the spectra precisely. At low mass, the

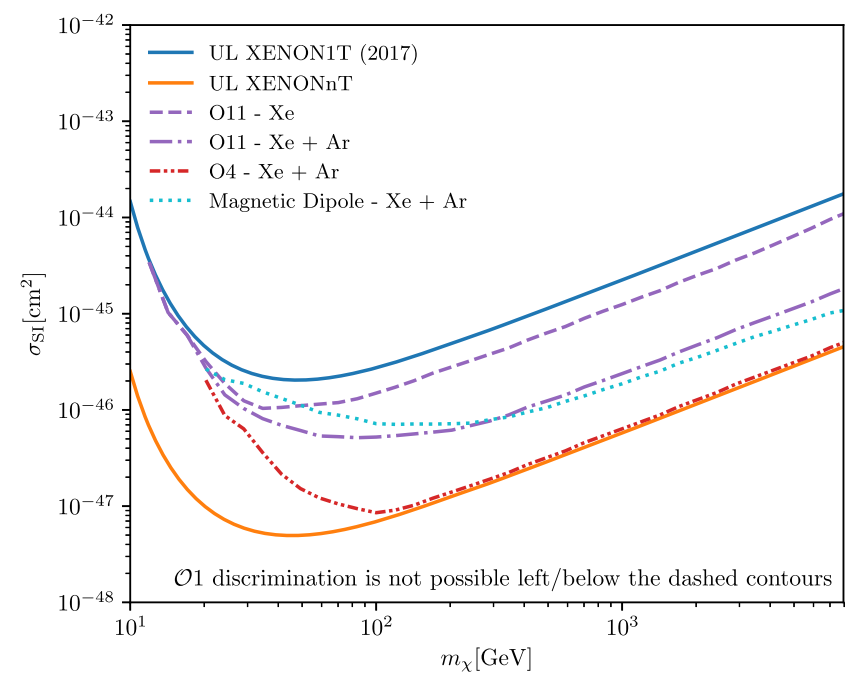

FIG. 3. Discriminability of DM interactions. To the left-below each broken line, it is not possible to discriminate an $\mathcal{O}_{1}$ signal (with the indicated cross section and DM mass) from the corresponding best-fit $\mathcal{O}_{4}, \mathcal{O}_{11}$, or magnetic dipole signal. Above-right of each broken line, such a discrimination is possible with at least $2 \sigma$ significance. Solid lines display 90\% C.L. for XENON1T-2017 and XENONnT. All lines include DM halo uncertainties. peak in the $\mathcal{O}_{11}$ spectrum falls below the threshold of the experiments; for both $\mathcal{O}_{1}$ and $\mathcal{O}_{11}$ the exponentially falling tail of the DM velocity distribution dominates the spectral shape [69], making discrimination impossible.

For magnetic dipole interactions, discrimination is also possible at high mass, given enough signal events. We note a "kink" in the boundary for magnetic dipole interactions around $m_{\chi} \sim 20 \mathrm{GeV}$. For large DM masses, the shortrange, spin-dependent, dipole-dipole contribution begins to dominate [48]. In this case, discrimination prospects are good with the inclusion of the spin-zero argon detector.

For the mock detectors we consider, SI interactions cannot be distinguished from Millicharged DM, which is not shown in Fig. 3. The recoil spectrum for Millicharged DM is similar to $\mathcal{O}_{1}$, but has an extra $E_{R}^{-2}$ suppression. This more rapidly falling recoil spectrum can be mimicked by an SI interaction with smaller DM mass. As demonstrated in Refs. [16,46], low-threshold semiconductor experiments are required to distinguish between the two interactions.

Finally, Fig. 4 shows, for various operators, the regions of parameter space where a closed contour for the DM mass would be possible at the $2 \sigma$ level. At large DM masses, the kinematics of the interaction mean that the recoil spectrum becomes independent of the DM mass, meaning that, to the right of the curves in Fig. 4, it is not possible to obtain an upper limit on $m_{\chi}[70,71]$. For $\mathcal{O}_{1}$ we show the regions for xenon only, as well as for xenon and argon combined without halo uncertainties to demonstrate the improvement in mass reconstruction when including a second detector. When the two detectors are combined, halo uncertainties

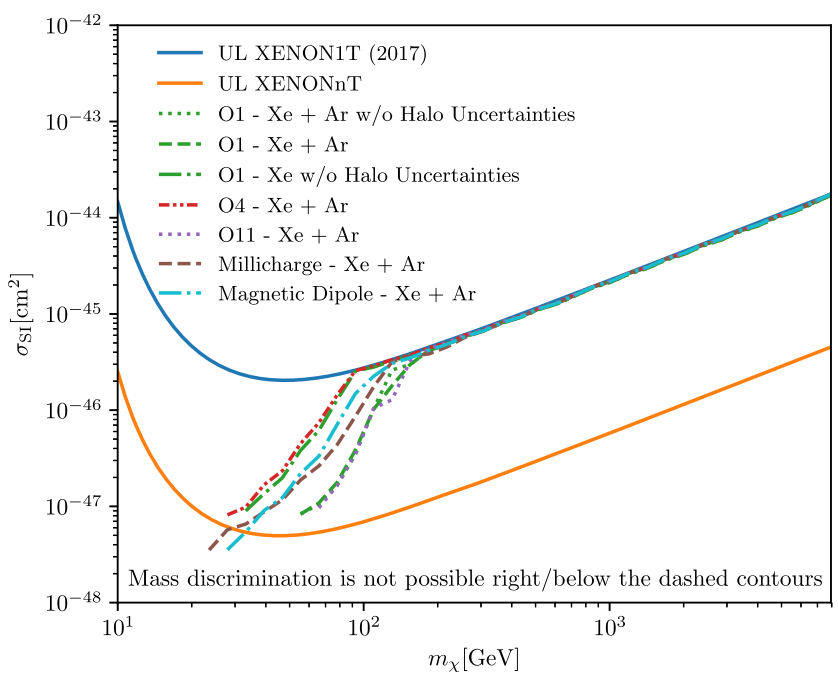

FIG. 4. Discriminability of the DM mass. To the right of each broken line, the DM mass is unbounded from above (at $2 \sigma$ ). Lines for other operators are mapped onto $\sigma_{\mathrm{SI}}$ by converting to an effective cross section and rescaled to match $\mathcal{O}_{1}$ at high masses. For $\mathcal{O}_{1}$, we also show constraints for the xenon-only case and when halo uncertainties are neglected. Solid lines display 90\% CL limits for XENON1T-2017 and XENONnT. 
make little difference to the mass discrimination, as changes in the velocity distribution affect the spectra in the two detectors differently [8]. Operator $\mathcal{O}_{4}$ contains the largest region in which the mass cannot be constrained due to the lack of signal in argon.

Even in the most optimistic case of cross sections just below the current bounds, it is not possible to pin down the DM mass for $m_{\chi} \gtrsim 100 \mathrm{GeV}$. Previous works have demonstrated, typically using a small number of benchmarks $[16,71,72]$, that DM mass reconstruction worsens for large masses; here, we have mapped out precisely where this mass reconstruction fails as a function of mass and cross section.

Discussion.-The methods introduced in this Letter allow us to efficiently characterize and visualize the phenomenological distinctiveness of direct DM signal models in infometric Venn diagrams, as shown in Fig. 2. Furthermore, these methods allow for an efficient exploration of the phenomenology of complex models, and hence allow us to make "benchmark-free" statements like those shown in Figs. 3 and 4. In Fig. 3 we see that ruling out nonstandard interactions is harder for light DM, while in Fig. 4 we see that we cannot pin down the DM mass for masses larger than $\sim 100 \mathrm{GeV}$. This leaves only a small region of parameter space-for $m_{\chi} \in[20,100] \mathrm{GeV}$ and cross sections a factor of a few below current bounds-in which the DM mass and interaction can both be constrained at the $2 \sigma$ level with near-future xenon and argon detectors. Such general statements would not have been possible without an efficient exploration of the Dark Matter parameter space, made feasible with the tools presented here. Third generation experiments such as DARWIN [64] will have a far greater sensitivity. More events would dramatically improve our ability to constrain different models, particularly for models at the current XENON1T bound.

This work paves the way for a more complete exploration of the direct detection parameter space and a deeper understanding of the complementarity between detectors. Future work should explore the possibility to discriminate between a wider range of interactions, beyond the subset of five we include here. In addition, the techniques we present may be used to optimize detector properties (target material, thresholds, etc.) in order to understand how operator discrimination can be improved at low DM mass.

Our benchmark-free method rests on the Euclideanized signal introduced in [31], and works for any Poisson (and hence Gaussian) likelihood function, as long as background uncertainties are sufficiently Gaussian. Euclideanized signals therefore provide a useful forecasting tool for a wide range of new-physics signals, including those in cosmology, indirect DM detection, and collider searches. As we have shown, using direct detection alone may not allow us to completely constrain the DM properties. Combining complementary information from other search strategies, coupled with new techniques for efficient forecasting, will provide essential guidance in the future of dark matter detection.

The authors thank Riccardo Catena for sharing fortran routines to calculate nuclear structure functions. In addition we thank the GAMBIT collaboration for extensive discussions on future direct detection instruments. We also thank Felix Kahlhoefer, Christopher McCabe, and Mauro Valli for very helpful comments on this manuscript. Finally, we thank the python scientific computing packages NUMPY [73] and SCIKIT-LEARN [74]. This research is funded by NWO through the VIDI research program "Probing the Genesis of Dark Matter" (680-47-532; T. E., C. W., B. K.).

*t.d.p.edwards@uva.nl

†.j.kavanagh@uva.nl

c.weniger@uva.nl

[1] G. Jungman, M. Kamionkowski, and K. Griest, Phys. Rep. 267, 195 (1996).

[2] G. Bertone, D. Hooper, and J. Silk, Phys. Rep. 405, 279 (2005)

[3] M. W. Goodman and E. Witten, Phys. Rev. D 31, 3059 (1985).

[4] A. K. Drukier, K. Freese, and D. N. Spergel, Phys. Rev. D 33, 3495 (1986).

[5] D. S. Akerib et al. (LUX Collaboration), Phys. Rev. Lett. 118, 021303 (2017).

[6] E. Aprile et al. (XENON Collaboration), Phys. Rev. Lett. 119, 181301 (2017)

[7] X. Cui et al. (PandaX-II Collaboration), Phys. Rev. Lett. 119, 181302 (2017).

[8] A. H. G. Peter, V. Gluscevic, A. M. Green, B. J. Kavanagh, and S. K. Lee, Phys. Dark Universe 5-6, 45 (2014).

[9] B. J. Kavanagh, M. Fornasa, and A. M. Green, Phys. Rev. D 91, 103533 (2015).

[10] M. Cahill-Rowley, R. Cotta, A. Drlica-Wagner, S. Funk, J. Hewett, A. Ismail, T. Rizzo, and M. Wood, Phys. Rev. D 91, 055011 (2015).

[11] A. Alves, A. Berlin, S. Profumo, and F. S. Queiroz, Phys. Rev. D 92, 083004 (2015).

[12] G. Bertone, N. Bozorgnia, J. S. Kim, S. Liem, C. McCabe, S. Otten, and R. Ruiz de Austri, J. Cosmol. Astropart. Phys. 03 (2018) 026.

[13] R. Catena, J. Cosmol. Astropart. Phys. 07 (2014) 055.

[14] M. I. Gresham and K. M. Zurek, Phys. Rev. D 89, 123521 (2014)

[15] V. Gluscevic and A. H. G. Peter, J. Cosmol. Astropart. Phys. 09 (2014) 040.

[16] V. Gluscevic, M. I. Gresham, S. D. McDermott, A. H. G. Peter, and K. M. Zurek, J. Cosmol. Astropart. Phys. 12 (2015) 057.

[17] F. Kahlhoefer and S. Wild, J. Cosmol. Astropart. Phys. 10 (2016) 032.

[18] R. Catena, J. Conrad, C. Dring, A. D. Ferella, and M. B. Krauss, arXiv:1706.09471.

[19] S. Baum, R. Catena, J. Conrad, K. Freese, and M. B. Krauss, Phys. Rev. D 97, 083002 (2018). 
[20] J. Fan, M. Reece, and L.-T. Wang, J. Cosmol. Astropart. Phys. 11 (2010) 042.

[21] A. L. Fitzpatrick, W. Haxton, E. Katz, N. Lubbers, and Y. Xu, J. Cosmol. Astropart. Phys. 02 (2013) 004.

[22] A. L. Fitzpatrick, W. Haxton, E. Katz, N. Lubbers, and Y. $\mathrm{Xu}$, arXiv:1211.2818.

[23] N. Anand, A. L. Fitzpatrick, and W. C. Haxton, Phys. Rev. C 89, 065501 (2014).

[24] J. B. Dent, L. M. Krauss, J. L. Newstead, and S. Sabharwal, Phys. Rev. D 92, 063515 (2015).

[25] R. Catena, J. Cosmol. Astropart. Phys. 09 (2014) 049.

[26] R. Catena and P. Gondolo, J. Cosmol. Astropart. Phys. 09 (2014) 045.

[27] R. Catena and P. Gondolo, J. Cosmol. Astropart. Phys. 08 (2015) 022.

[28] The code associated with this Letter is available at https:// github.com/tedwards2412/benchmark_free_forecasting/.

[29] G. Cowan, K. Cranmer, E. Gross, and O. Vitells, Eur. Phys. J. C 71, 1554 (2011); 73, 2501(E) (2013).

[30] S. S. Wilks, Ann. Math. Stat. 9, 60 (1938).

[31] T. D. P. Edwards and C. Weniger, arXiv:1712.05401.

[32] J. Venn, London, Edinburgh, Dublin Philos. Mag. J. Sci. 10, 1 (1880).

[33] Please see the Supplemental Material at http://link.aps.org/ supplemental/10.1103/PhysRevLett.121.181101 for technical details on implementing the Euclideanised signal method and modeling the Dark Matter signal.

[34] D. G. Cerdeno and A. M. Green, in Particle Dark Matter: Observations, Models and Searches, edited by G. Bertone (Cambridge University Press, Cambridge, England, 2010), pp. 347-369.

[35] S. Chang, A. Pierce, and N. Weiner, J. Cosmol. Astropart. Phys. 01 (2010) 006.

[36] R. Catena, J. Cosmol. Astropart. Phys. 07 (2015) 026.

[37] B. J. Kavanagh, Phys. Rev. D 92, 023513 (2015).

[38] E. Del Nobile, G. B. Gelmini, and S. J. Witte, Phys. Rev. D 91, 121302 (2015).

[39] E. Del Nobile, G. B. Gelmini, and S. J. Witte, J. Cosmol. Astropart. Phys. 02 (2016) 009.

[40] We neglect the effects of operator mixing [41,42], which require us to specify the structure of the dark sector.

[41] F. D'Eramo, B. J. Kavanagh, and P. Panci, J. High Energy Phys. 08 (2016) 111.

[42] F. Bishara, J. Brod, B. Grinstein, and J. Zupan, arXiv: 1708.02678

[43] D. G. Cerdeo et al., J. Cosmol. Astropart. Phys. 07 (2013) 028; 09 (2013) E01(E).

[44] Note, however, that because the DM is still nonrelativistic, the effects of light mediators can be incorporated into the NREFT by including the appropriate propagator $[45,46]$.

[45] M. Cirelli, E. Del Nobile, and P. Panci, J. Cosmol. Astropart. Phys. 10 (2013) 019.

[46] F. Kahlhoefer, S. Kulkarni, and S. Wild, J. Cosmol. Astropart. Phys. 11 (2017) 016.
[47] C. M. Ho and R. J. Scherrer, Phys. Lett. B 722, 341 (2013).

[48] E. Del Nobile, G. B. Gelmini, P. Gondolo, and J.-H. Huh, J. Cosmol. Astropart. Phys. 06 (2014) 002.

[49] S. D. McDermott, H.-B. Yu, and K. M. Zurek, Phys. Rev. D 83, 063509 (2011).

[50] N. Fornengo, P. Panci, and M. Regis, Phys. Rev. D 84, 115002 (2011).

[51] P. Panci, Adv. High Energy Phys. 2014, 681312 (2014).

[52] K. Sigurdson, M. Doran, A. Kurylov, R. R. Caldwell, and M. Kamionkowski, Phys. Rev. D 70, 083501 (2004); 73, 089903 (2006).

[53] T. Banks, J.-F. Fortin, and S. Thomas, arXiv:1007.5515.

[54] J. Bagnasco, M. Dine, and S. D. Thomas, Phys. Lett. B 320, 99 (1994).

[55] T. Banks, J. D. Mason, and D. O’Neil, Phys. Rev. D 72, 043530 (2005).

[56] V. Barger, W.-Y. Keung, and D. Marfatia, Phys. Lett. B 696, 74 (2011).

[57] E. Del Nobile, C. Kouvaris, P. Panci, F. Sannino, and J. Virkajarvi, J. Cosmol. Astropart. Phys. 08 (2012) 010.

[58] B. J. Kavanagh and T.D.P. Edwards, WIMpy_NREFT v1.0, Doi: https://github.com/bradkav/WIMpy_NREFT (2018).

[59] Note that the operator normalizations in [45] and [23] differ.

[60] R. Catena and B. Schwabe, J. Cosmol. Astropart. Phys. 04 (2015) 042.

[61] J. I. Read, J. Phys. G 41, 063101 (2014).

[62] A. M. Green, J. Phys. G 44, 084001 (2017).

[63] D. S. Akerib et al. (LZ Collaboration), arXiv:1509.02910.

[64] J. Aalbers et al., J. Cosmol. Astropart. Phys. 11 (2016) 017.

[65] G. Plante (XENON Collaboration), The XENONnT Project, https://conferences.pa.ucla.edu/dm16/talks/plante.pdf.

[66] D. S. Akerib et al. (LUX-ZEPLIN Collaboration), arXiv: 1802.06039 .

[67] C. E. Aalseth et al., Eur. Phys. J. Plus 133, 131 (2018).

[68] Liquid noble detectors typically do not have sensitivity to DM particles lighter than a few $\mathrm{GeV}$, so we restrict our attention to $m_{\chi}>10 \mathrm{GeV}$ in the current work.

[69] J. D. Lewin and P. F. Smith, Astropart. Phys. 6, 87 (1996).

[70] A. M. Green, J. Cosmol. Astropart. Phys. 08 (2007) 022.

[71] A. M. Green, J. Cosmol. Astropart. Phys. 07 (2008) 005.

[72] J. L. Newstead, T. D. Jacques, L. M. Krauss, J. B. Dent, and F. Ferrer, Phys. Rev. D 88, 076011 (2013).

[73] T. E. Oliphant, Guide to NumPy, 2nd ed. (CreateSpace Independent Publishing Platform, USA, 2015).

[74] F. Pedregosa, G. Varoquaux, A. Gramfort, V. Michel, B. Thirion, O. Grisel, M. Blondel, P. Prettenhofer, R. Weiss, V. Dubourg, J. Vanderplas, A. Passos, D. Cournapeau, M. Brucher, M. Perrot, and E. Duchesnay, J. Mach. Learn. Res. 12, 2825 (2011). 